\title{
INVESTIGACIONES
}

\section{La práctica en la formación de profesores: tensiones y posibilidades en la experiencia de aprender a enseñar}

\author{
The practicum in preservice teacher education programs: strengths and weaknesses
} in the experiences of learning to teach

\section{Prática na formação de professores: tensões e possibilidades na experiência de aprender a ensinar}

\author{
Tom Russell \\ Queen's University, Canada \\ Correo electrónico: russellt@ queensu.ca
}

\begin{abstract}
RESUMEN
Este artículo identifica tensiones y posibilidades sobre dos formas de concebir la práctica en los programas de formación de profesores: la inducción gradual a la responsabilidad en la enseñanza versus una aproximación que refiere a una rápida inducción en los espacios de práctica con una mínima introducción y preparación formal. Se plantea como propósito hacer un llamado de atención a la reticencia extrema por parte de los formadores de docentes a preguntarse sobre las premisas implícitas que sustentan sus enfoques sobre la formación práctica, explorando aproximaciones alternativas que den mayor relevancia al papel de la experiencia de aquellos que participan en el proceso formativo. Este hecho permite entender por qué el cambio es más una promesa que una acción concreta.
\end{abstract}

Palabras clave: prácticas, experiencia, aprendizaje de la enseñanza.

\begin{abstract}
This article identifies strengths and weaknesses of two practicum assumptions in teacher education: the traditional gradual introduction to teaching responsibility versus a rapid introduction approach that would place teacher candidates into an extended teaching practicum with minimal formal introduction and preparation. The purpose of this article is to call attention to what appears to be an extreme reluctance on the part of teacher educators to explore the assumptions underlying a traditional practicum approach. Teacher educators tend not to ask if there might be alternative approaches to preservice teacher education that could make greater academic and practical use of experience in the introduction to the teaching profession. This provides a way to understand why the change is more a promise than a reality.
\end{abstract}

Key words: practicum, experience, learn to teach.

\section{RESUMO}

Identificam-se tensões e possibilidades em duas formas de conceber a prática em programas de formação de professores: a indução gradual de responsabilidade no ensino contra uma aproximação que se refere a uma rápida indução em espaços de prática com introdução mínima e preparação formal. Apresenta-se com o objetivo de fazer um chamado de atenção à reticência extrema dos formadores de professores para questionar sobre as premissas implícitas que sustentam seus enfoques sobre a formação prática, explorando aproximações alternativas que dão maior importância ao papel da experiência de que aqueles que participam do processo formativo. Isto como uma maneira de entender por que a transformação é mais uma promessa do que uma ação concreta.

Palavras-chave: prática, experiencia, aprendizagem do ensinar. 
Lo habitual de la práctica de la enseñanza no compensa su carácter irreflexivo; el futuro profesor no está demandado a comparar, analizar, y seleccionar desde diversas posibilidades. El riesgo es, claramente, que la práctica de enseñanza puede ser una simple exposición de los estudiantes a los estilos de trabajo de sus profesores. El valor de la práctica es algo reconocido por muchos de los que han participado en ella, pero hay poca referencia que muestre como se va más lejos del individualismo y lo tradicional. Cualquier parecido con la realidad es mera coincidencia con lo que pase en nuestras clases.

(Lortie, 1975: 71)

Una característica básica de los programas de formación de profesores es la introducción gradual de los futuros profesores a experiencias en clases y escuelas. En dichos programas las prácticas ocupan dos o tres años, siendo común comenzar las experiencias prácticas con visitas a las escuelas y aulas con el fin de observar, tutorear y asistir a los docentes. Gradualmente el futuro profesor, en algún momento, asumirá la clase a tiempo completo; asignaciones de prácticas cortas que usualmente terminan con una mayor cantidad de tiempo en sala, hacia el fin del programa. Recientemente, hemos visto cambios referidos al aumento de tiempo invertido en los lugares de práctica, pero este tipo de cambio no ha sido acompañado por una mayor cantidad de análisis fundamentales del rol de la experiencia en el aprendizaje de la enseñanza. Las conclusiones a las que llegó Lortie (1975) hace 25 años suenan cada vez más verdaderas. Las críticas a la formación de profesores en términos de tensión entre teoría y práctica no han desaparecido y no han sido resueltas. Los vínculos estructurales entre teoría y práctica a menudo no están presentes en las experiencias de práctica en la mayoría de los programas de formación de profesores.

\section{EL CONTEXTO PARA EL CAMBIO EN LOS PROGRAMAS DE FORMACIÓN DOCENTE}

Pareciera que los llamados a mejorar nuestras escuelas no tienen fin, y lo mismo puede decirse de los llamados a mejorar la formación de profesores. En esta sección, tomo las perspectivas de Sarason, Bruner, y Kessels y Korthagen para mostrar las maneras en que las aproximaciones tradicionales a la práctica en la formación inicial docente no han sido capaces de examinar las premisas subyacentes acerca de cómo la experiencia práctica puede contribuir al proceso de aprender a enseñar.

Tanto en la escuela como en las universidades, los procesos de cambio parecen ser similares; los cambios realmente fundamentales son escasos (Sarason, 1971). Cuando Sarason (1996) revisó y expandió su análisis sobre el cambio en la escuela (1971), concluyó que los cambios en las condiciones para aprender deben ocurrir en paralelo tanto para los estudiantes como para los profesores. Asimismo, identificó que la relación profesoralumno como "el que pregunta y el que responde" es la principal característica que requiere ser cambiada. Sostengo que el patrón "profesor pregunta-estudiante responde" está en el corazón de la aproximación "teoría primero, práctica después" que caracteriza no sólo el modo en que los profesores enseñan, sino también el modo en que aprenden a enseñar. En The Case for Change: Rethinking the Preparation of Educators (Sarason, 1993) resume su postura con los siguientes puntos: 
El objetivo principal de la educación es alimentar el sentido de descubrimiento y crecimiento en los estudiantes y profesores (p. 138).

En el contexto de las salas de clases y escuelas hay obstáculos enormes para llevar a cabo acciones consistentes con el objetivo principal (p. 138).

Los que buscan convertirse en educadores tienen a su haber un capital importantísimo: han pasado años como "aprendices" en salas de clase (p. 139).

Sarason explica la necesidad de preparar a los futuros docentes para lidiar con los obstáculos que se presentan en el proceso de incentivar el descubrimiento y el crecimiento, y su tercer punto indica la necesidad de hacer algo con respecto a las experiencias previas en la sala de clases de los futuros profesores. Hacerse cargo de las experiencias pasadas y presentes en la educación raramente ha sido una característica central en el proceso de aprender a enseñar.

La importancia de encargarse de las experiencias previas en la escuela de los futuros profesores se apoya en una perspectiva relacionada con Bruner (1996), quien usa el término pedagogía popular para referirse a aquello que se ha arraigado profundamente sobre la pedagogía, formado desde las percepciones de la enseñanza:

Al teorizar sobre la práctica de la educación en la sala de clases (...) te conviene tomar en consideración las teorías ya instaladas en aquellos involucrados en la enseñanza y aprendizaje. Porque cualquier innovación que (...) quieras introducir tendrá que competir, reemplazar o modificar las teorías populares que ya están guiando el actuar tanto de profesores como de estudiantes. (Bruner, 1996: 44).

La mayoría de los que deciden estudiar pedagogía llegan a los programas de formación con el supuesto de que son "vasijas vacías" en lo que respecta a la práctica de la enseñanza. Esta trágica suposición es una consecuencia natural de 15,000 horas de colegio y experiencias de salas de clase que no han hecho ninguna consideración explícita respecto a cómo los estudiantes han sido enseñados. Lo que Bruner ve como "teorías populares" acerca de la pedagogía son posiblemente tanto o más poderosas que las concepciones previas de los estudiantes acerca de fenómenos científicos, concepciones que han demostrado ser altamente persistentes fuera de la sala de clases de ciencia, a pesar de los mejores esfuerzos de sus profesores.

Las pedagogías populares (...) reflejan una variedad de supuestos acerca de los niños: pueden ser vistos como voluntariosos y en necesidad de corrección; como inocentes y en necesidad de ser protegidos de una sociedad vulgar; como necesitados de habilidades a ser desarrolladas sólo a través de la práctica; como vasijas vacías a ser llenadas con conocimiento que sólo los adultos proveen; como egocéntricos y en necesidad de ser socializados. Creencias populares como las de este tipo, expresadas por gente común o por "expertos", casi nunca quieren ser deconstruidas si ello involucra develar sus implicancias. Ya sea que estas visiones sean "correctas" o no, su impacto en las actividades de enseñanza puede ser enorme. (Bruner, 1996: 49).

Se puede reconocer que los que entran a los programas de formación inicial docente vienen cargados de teorías populares acerca de la pedagogía, y sin embargo, los elementos y secuencia tradicional de las prácticas no logran lidiar con esas teorías.

Quizás el desafío más evidente para cambiar en cualquier ámbito de la formación de profesores es nuestro desconocimiento individual y colectivo de cualquier cosa a la que vayamos a cambiar. A pesar de las numerosas diferencias entre profesores, las grandes 
similitudes al entregar contenidos y construir pruebas, nos dejan con escaso espacio para la imaginación y un reducido número de alternativas posibles. "La innovación genuina genera incompetencia" (MacDonald, 1975: 11) es una frase que apunta hacia el problema. Escuchamos incesantes llamados a desarrollar aproximaciones constructivistas de aprendizaje, o acerca del potencial de las comunidades de aprendizaje, sin embargo no tenemos la experiencia de crear, mantener o defender tales aproximaciones. La "experiencia" juega un rol en todo esto; la mayoría de las personas está de acuerdo en que el aprendizaje desde la experiencia es tremendamente poderoso y que perdura más que el aprendizaje que pueda ocurrir porque alguien dijo algo.

Korthagen y Kessels (1998) han provisto de una importante contribución a este desafío, escribiendo en el contexto de reforma en la formación de profesores. Construyen su argumento sobre la base de un contraste fundamental entre episteme y phronesis. La Episteme, estrechamente asociada a la ya familiar epistemología, es la base sobre la que se afirma el conocimiento; es el fundamento de la universidad, y la institución del libro de texto es su corporeización más visible. La phronesis, relacionada a la percepción, es generalmente foránea al contexto de la escuela y la universidad. Kessels y Korthagen (1998) han argumentado que la phronesis guarda un potencial considerable para enfrentar el eterno problema de teoríapráctica que es tan frecuentemente ignorado por la perspectiva de la episteme.

\begin{abstract}
Alguien podría reconocer la importancia de periodos de práctica progresivas en los programas de formación de profesores y aun así perder completamente de vista el punto de la phronesis. De hecho, muchos formadores de profesores que rescatan el valor de la experiencia práctica terminan, sin embargo, trabajando sobre la base de una concepción epistémica de conocimiento; tienen dificultades con la brecha entre teoría y práctica, se preocupan y quedan perplejos ante problemáticas de transferencia, y buscan de qué manera poder conectar mejor con los conocimientos previos de los estudiantes... El punto de la phronesis es que el saber que necesita un estudiante es de carácter perceptivo antes que conceptual. Por lo tanto, es necesariamente interno en el estudiante, está en la experiencia del estudiante en lugar de afuera, en alguna forma conceptual. Es absolutamente subjetivo... y por tanto hay nada o muy poco que transmitir, y al contrario, hay muchísimo por explorar. Y la tarea del formador de profesores es ayudar al profesor en formación a explorar y refinar sus percepciones. Esto requiere de procesos muy bien organizados en los cuales los futuros profesores tengan la oportunidad de reflexionar sistemáticamente sobre los detalles de sus experiencias prácticas, bajo la guía del formador de profesores- tanto en seminarios grupales como en la supervisión individual (Kessels \& Korthagen, 1996: 21; énfasis añadido).

El peligro del énfasis en un conocimiento procedimental en la formación de profesores es que los estudiantes aprenden mucho métodos, protocolos y estrategias para diversas situaciones pero no aprenden a descubrir, en las situaciones específicas del día a día en la enseñanza, qué métodos y estrategias usar (Korthagen \& Kessels, 1998: 7, énfasis añadido).
\end{abstract}

Importantes reflexiones acerca de la utilidad de una perspectiva de phronesis en la formación de profesores son presentadas en Korthagen et al. (2001), aunque un entendimiento profundo de una perspectiva poco familiar como esta resulta difícil de no haber de por medio una experiencia personal significativa en el intento por usar esta perspectiva en el propio contexto de práctica.

En las secciones que vienen describiré una propuesta innovadora que ha sido probada en la Universidad de Queen's y exploraré la problemática de los supuestos acerca del 
proceso de aprender a enseñar al interior de las prácticas profesionales. Luego contrastaré la introducción gradual y la inmersión rápida a la experiencia para destacar la naturaleza no revisada de esos supuestos.

\section{ESTRUCTURA DEL PROGRAMA DE FORMACIÓN DOCENTE EN LA UNIVERSIDAD DE QUEEN'S}

Tal como se registró en otras publicaciones (Russell, 1999, 2000), la Facultad de Educación de la Universidad de Queen's transformó la estructura del programa de formación inicial de profesores en 1997-1998, luego de dos años de planificación, de consulta y de un proyecto piloto que involucró la participación de 60 voluntarios. En los dos años iniciales (1997-1999), todos los estudiantes comenzaron sus experiencias prácticas el primer día de clases, luego de una introducción y orientación de una semana en la universidad. La experiencia duraba de Septiembre a Diciembre, en un periodo de 16 semanas interrumpidas por un regreso de dos semanas a la universidad, en la mitad del proceso.

En términos muy sucintos, funcionó, y funcionó muy bien; con unas pocas excepciones inevitables. Los futuros profesores estaban muy conformes. Muchos profesores colaboradores también estaban contentos con la experiencia, pero muchos otros no. Una preocupación para algunos era el sentirse incómodos al ser observados durante el primer día. Otra preocupación más compartida, parecía ser la opinión de que la universidad debía enseñarles a los futuros profesores cómo enseñar antes de enviarlos a la práctica. La mayoría de mis colegas parecían estar de acuerdo con este último grupo de profesores de escuela: la teoría debía preceder a la práctica. Desafortunadamente, y siguiendo el patrón habitual en educación, la visión de los estudiantes fue muy poco valorada. Desde 1999, "pequeñas" modificaciones al programa han seguido erosionando la premisa inicial de que aprender de la experiencia puede y debe ser una meta y método fundamental del programa.

La experiencia personal de haber visto un cambio radical exitoso en sus inicios, el que fue abandonado ante la total ausencia de consideraciones de las premisas subyacentes a la práctica, me impulsa a explorar esos supuestos como una preocupaciones permanente acerca de la incapacidad de las universidades para alcanzar la reforma de sus programas de formación docente (Goodlad, 1990; Sarason, 1993). Los ejemplos usados en los siguientes análisis surgen del autoestudio de mi propia enseñanza de profesores en pregrado, y de estudios de caso acerca de eventos en mi universidad.

Localizada en la provincia Canadiense de Ontario -que alberga a un tercio de la población del país- la Universidad de Queen's ha experimentado reducciones anuales en el financiamiento gubernamental durante los últimos 15 años. Entre los años 1995 al 2003, un gobierno provincial conservador legisló profundos cambios estructurales al financiamiento de servicios médicos, educacionales y sociales, junto con un programa general de reducción de impuestos. En el año escolar 2002-2003, el tradicional quinto año de enseñanza secundaria fue removido. La profesión docente fue calificada como un "grupo de interés especial" y una insatisfacción profesional y una perturbación en el clima escolar surgieron como consecuencias naturales de las constantes críticas del gobierno. Mientras que muchas provincias canadienses han modificado su formación docente a programas de dos años de duración, en Ontario se mantienen en los niveles de ocho a diez meses, con un requisito mínimo de 4 días de experiencia práctica. 
El patrón habitual para la formación docente en Ontario involucra la obtención de una Licenciatura en Educación (B.Ed. en Inglés), en un programa que abarque un año académico luego de la previa obtención de una licenciatura en alguna disciplina. La cohorte de formación docente 2003-2004 en Queen’s comenzó al inicio de Septiembre y terminó a fines de Abril. Más de 700 candidatos fueron divididos más o menos equitativamente en enseñanza primaria y secundaria; alrededor de un tercio se había matriculado en un programa concurrente (que abarca la formación disciplinar y pedagógica) desde el inicio de su formación de pregrado tanto en Queen’s como en la cercana Universidad de Trent. Los cursos ofrecidos están en las categorías de Currículum, Focus Track, Fundamentos, Estudios Educacionales y Estudios Profesionales.

El primero de los dos semestres comienza en Septiembre con tres semanas de clases previas a las cinco primeras semanas de práctica en una escuela asociada; dos semanas más de clase y cinco semanas más de práctica en la misma escuela completan el primer semestre. El segundo semestre comienza con siete semanas de clases y continúa con dos bloques de práctica: una "práctica alternada", generalmente en un contexto educativo no escolar, y relacionado a la categoría de cursos Focus Track, y tres semanas de práctica adicionales, frecuentemente en la misma escuela asociada a la que se asistió durante el primer semestre. El programa concluye con dos semanas finales de clases, una incluye un "seminario de egreso" en el que se comparte un portafolio de desarrollo profesional con el resto del curso.

El modelo de Escuela Asociada, introducido durante la reestructuración del programa en 1997-1998, requiere la asignación de estudiantes en grupo, en lugar de individualmente. Cada grupo es apoyado durante la práctica por un representante de la Facultad, el que puede ser un académico de planta, aunque generalmente es un profesor o director jubilado, o un candidato a doctor en Educación. Los grupos de educación primaria incluyen entre tres a ocho personas, mientras los de educación secundaria de dos a doce, y en ocasiones más. Por lo mismo, el representante de la Universidad ofrece apoyo y supervisión en todo el rango de asignaturas escolares. A los estudiantes se les pide que se reúnan tres horas semanales para discutir experiencias y asuntos relacionados a equidad, excepcionalidad, investigación acción y desarrollo profesional. En la próxima sección de este escrito compararé los supuestos que sustentan la estructura de primero experiencia, versus la estructura de primero teoría.

\section{COMPARANDO SUPUESTOS ALTERNATIVOS ACERCA DE LA PRÁCTICA EN LA FORMACIÓN INICIAL DE PROFESORES}

Al interior de la ya habitual aproximación de inducción gradual a la experiencia práctica se encuentran varios supuestos y perspectivas interrelacionadas, las que podrían ser expresadas en los siguientes términos:

a. Los que están aprendiendo a enseñar requieren de una extensa preparación para asumir las responsabilidades de un docente. Sus muchos años de experiencia como estudiantes sirven de poco para guiar la experiencia de enseñar.

b. Los que están aprendiendo a enseñar pueden ser ayudados en su preparación para las responsabilidades de la enseñanza por medio de la observación y colaboración en la sala de clases. Tales actividades tienen mayor valor cuando van acompañadas de orientaciones para 
la observación y para el reporte de sus actividades como observador o asistente.

c. Los que están aprendiendo a enseñar son capaces de desarrollar mayor control sobre sus comportamientos docentes a medida que logran ver con mayor detalle, por medio de sus experiencias de observación, las complejidades de los procesos de aprendizaje grupal e individual.

$\mathrm{Al}$ interior de la no tan tradicional aproximación de inducción rápida a la experiencia práctica se encuentran varios supuestos y perspectivas interrelacionadas, las que podrían ser expresadas en los siguientes términos:

a. Los que están aprendiendo a enseñar tienen una vasta experiencia con lo que ocurre en una sala de clases, pero se ha hecho poco para analizar e interpretar esa experiencia. Han tenido muy poco acceso al modo en que los profesores planifican y piensan acerca de su trabajo.

b. Los que están aprendiendo a enseñar progresan con mayor rapidez cuando parten enseñando clases completas, que les permiten experimentar el modo en que un profesor necesita pensar acerca de los estudiantes a los que se les está enseñando y el modo en que se les enseña.

c. Los que están aprendiendo a enseñar necesitan ver que enseñar una clase completa es drásticamente diferente a sentarse a observar, o a ayudar en la sala de clases.

Por un asunto de conveniencia, etiqueto estos supuestos como "teoría primero, práctica después" (introducción gradual a la práctica) y "experiencia primero, comprensión después" (introducción rápida a la práctica) respectivamente. En la siguiente sección profundizo en esta discusión al mostrar una serie de asuntos asociados a estas dos aproximaciones a la práctica.

\subsection{TEORÍA PRIMERO, PRÁCTICA DESPUÉS}

Esta sección comienza con algunos comentarios acerca de la tradición de introducción gradual a la enseñanza y la experiencia práctica. Luego continúa con puntos relevantes sobre la relación entre práctica y reforma de la formación docente, explorando dos problemáticas particulares: los formadores de profesores frecuentemente son ambivalentes respecto a las escuelas en las que ocurre la práctica; el supuesto de los formadores de profesores de que sumar una práctica reflexiva a una aproximación de "teoría primero" puede ser contradictorio.

\subsubsection{La tradición de introducción gradual}

La introducción gradual es apropiada y comprensiblemente utilizada en campos en los que el principiante no tiene experiencia alguna. Un aprendiz de médico no entra directamente a una cirugía, del mismo modo en que un futuro abogado no entra directamente a un tribunal. En estas profesiones hay muchísimas cosas por aprender que son completamente desconocidas. Se puede argumentar que el caso de educación es bastante distinto, puesto que todos los candidatos a profesor ya vienen con más de 15,000 horas de experiencia observando profesores hacer su trabajo. Para la mayoría de las personas, esa experiencia nunca ha sido examinada, y sin embargo es lo que lleva al desarrollo de aquello que Bruner ha denominado "teorías populares" de la pedagogía.

La práctica de introducción gradual es bastante cuestionable en el campo de la enseñanza debido a que las imágenes (adquiridas en contexto) son mucho más poderosas 
que las palabras (escuchadas fuera del contexto). Tanto los formadores de profesores como los futuros docentes parecen no reconocer esto como un asunto relevante, posiblemente debido a que las prácticas de enseñanza tradicional han depositado una enorme confianza en el poder de la palabra hablada.

La tradición de la introducción gradual a la experiencia persiste en parte porque resulta familiar mientras que las alternativas son desconocidas y representan un riesgo. También se mantienen porque aquellos que ya aprendieron a enseñar quieren ser de ayuda a los que están recién comenzando. Al mismo tiempo, la formación de profesores frecuentemente alberga un complejo y no analizado temor, a que los estudiantes serán “contaminados” por las actuales prácticas de la escuela. De ese modo, los formadores de profesores asumen, o al menos esperan, que nuestras palabras logren "inmunizar" a los profesores principiantes de las malas prácticas que persisten en la escuela.

\subsubsection{La ambivalencia de los formadores de profesores sobre el centro de práctica}

Los formadores de profesores son conocidos por su criticidad de las prácticas "tradicionales" de enseñanza en la escuela. Este es uno de los muchos elementos que hacen que una genuina alianza escuela-universidad sea algo tan difícil de alcanzar. Los formadores saben que hay llamados para que las escuelas cambien, del mismo modo que saben que muchos candidatos a profesores no verán en sus prácticas mucho de lo que se les recomienda desde las clases de pedagogía. Las instituciones formadoras de profesores frecuentemente defienden aproximaciones asociadas a frases como "constructivismos", "aprendizaje cooperativo en grupos pequeños", "aprendizaje basado en problemas" y "enseñar de acuerdo a las necesidades individuales de los estudiantes". Aunque tales prácticas son muy escasas en las escuelas, nuestras universidades no son precisamente conocidas por criticar sus propias prácticas. Tanto la escuela como la universidad continúan mostrando una gran confianza en la enseñanza por "transmisión". La introducción gradual a la práctica resulta consistente con las tradiciones de una enseñanza y aprendizaje basados en la transmisión: primero el profesor te dice lo que tienes que saber, y luego tienes que ir y ponerlo en práctica.

\subsubsection{Añadir "prácticas reflexivas" a la "teoría primero" puede ser un error}

“Queremos temáticas relevantes, no sólo palabras bonitas”, fueron las palabras de un profesor en formación en la Universidad de Queen's, palabras que expresaban la expectativa respecto a las clases formales luego de 14 semanas en una práctica de inmersión. Las experiencias prácticas habían elevado las expectativas: las palabras y buenas intenciones ya no eran suficientes para satisfacer lo que se esperaba de las clases. La experiencia genera más que sólo preguntas; también genera un filtro que permite distinguir rápidamente entre qué es práctico y realista, y que no lo es, entre lo que funcionará en una sala de clases real y lo que no.

Las exhortaciones a ser un profesional "crítico y reflexivo" pueden no ser más que palabras bonitas usadas por académicos que no demuestran ser profesionales de tales características. Si los profesores en formación han visto poca evidencia de profesores en la escuela que sean críticos y reflexivos acerca de sus prácticas, entonces necesitan poder ver evidencia de ello en las acciones de sus propios formadores en la universidad. La reflexión tiene relación directa con la experiencia, y si las clases de pedagogía no son 
ricas en experiencia, entonces ¿dónde están las posibilidades de reflexión? La reflexión requiere ser enseñada y modelada. La simple inclusión de exhortaciones al interior de una aproximación tradicional de "teoría primero", termina dirigiendo más atención a lo que falta en lugar de ayudar a los estudiantes a ser críticos de las prácticas. Segall (2002) presenta una potente argumentación para tender un puente entre teoría y práctica por medio del uso de las experiencias como base para el aprendizaje en las clases de educación.

\subsection{EXPERIENCIA PRIMERO, LUEGO COMPRENSIÓN}

Esta sección comienza con algunos comentarios acerca de cómo los profesores de todos los niveles educativos no están familiarizados con un aprendizaje enraizado en la experiencia, en lugar de recuentos organizados lógicamente acerca de las experiencias de otras personas (el currículum escolar). Luego continúa con varias perspectivas acerca de la experiencia de inmersión en la práctica.

\subsubsection{El desconocido terreno del aprendizaje desde la experiencia}

Proveer primero la experiencia a aquellos que están aprendiendo a enseñar no surge fácilmente, y pareciéramos tener una resistencia natural a enfocar los programas de formación en un aprendizaje desde la experiencia. Virtualmente, todos los formadores de profesores comienzan a trabajar enseñando del mismo modo en que ellos fueron enseñados. La resistencia a tener una experiencia práctica al inicio de un programa de formación va más allá de nuestra poca familiaridad con el aprendizaje desde la experiencia en un contexto escolar o académico. La predisposición a "ayudar" y hacer de las primeras experiencias de enseñanza algo "más fácil" nos viene como algo natural a los adultos. Nuestra cultura tiene una tradición de larga data en el intentar hacer la vida más fácil para la siguiente generación, tanto en la relación padres-hijos como en la de profesor-estudiante. Esta predisposición sostiene además la fe que los formadores tienen en el conocimiento proposicional, a pesar de las décadas de evidencia que demuestra que la teoría no se traduce directamente en una práctica.

Colectivamente, los formadores de profesores parecen tener un compromiso fundamental con la importancia de primero generar lazos afectivos con el sitio de la formación docente y un lazo menos poderoso con el lugar de práctica aparece después. Aun cuando los que están aprendiendo a enseñar siempre reportan que la práctica es el elemento más importante del programa, esas experiencias de práctica rara vez llegan a tener preeminencia en los tiempos usados durante las clases de educación. En este sentido, los formadores de profesores parecen guardar un cierto temor subconsciente a que los estudiantes lleguen a "enamorarse" a tal punto de la práctica que pierdan todo interés en lo "teórico".

\subsubsection{Comentarios acerca de la aproximación de "nadar o morir" en la práctica}

Los siguientes comentarios fueron seleccionados de aquellos ofrecidos por un grupo de candidatos del programa de la Universidad de Queen’s del año 1996.

Un acuerdo firmado con la Universidad de Waterloo generó la oportunidad para que un pequeño grupo de futuros profesores de ciencias pudieran enseñar durante cuatro meses antes de comenzar sus cursos de educación. Mientras que muchos de ellos mencionaron el shock 
Estudios Pedagógicos, vol. XL, Número Especial 1: 223-238, 2014

LA PRÁCTICA EN LA FORMACIÓN DE PROFESORES: TENSIONES Y POSIBILIDADES EN LA EXPERIENCIA DE

APRENDER A ENSEÑAR

que les produjo la rápida inmersión en la enseñanza, también destacaron con especial énfasis los aspectos motivadores de la experiencia, incluyendo la generación de preguntas a las que se esperaría que los cursos de educación pudieran dar una respuesta, al menos preliminarmente.

\begin{abstract}
Ahora sé de muchos libros que tendré que leer, preguntas que necesito hacer y cosas que debo aprender, pero no fue sino hasta que estuve enseñando durante una semana que pude realmente darme cuenta de qué era lo que necesitaba preguntar, o en qué necesitaba ayuda. Así que el método de "nadar o morir" es excelente... Antes de enseñar, no tenía idea en qué áreas necesitaría ayuda. Luego de la experiencia, tenía una lista de casi un metro de largo con preguntas. Andrea. (Featherstone, Munby y Russell, 1997: 87-88).

Lo central es querer aprender y estar dispuesto a asumir riesgos y disfrutar o sufrir las consecuencias, sean las que sean. Los estudiantes son generalmente muy transparentes y te harán saber muy prontamente cómo te está yendo... Con todo, creo que la experiencia fue genial. No sólo metí los pies al agua, me empapé! Y valió la pena. La mejor manera de aprender algo es lanzarse directo a ello. Steph. (Featherstone, Munby y Russell, 1997: 91).

Es imposible enseñarle a alguien a enseñar; sólo puedes guiarlo... Tengo experiencias, tanto malas como buenas, pero puedo mejorar a través de mi aprendizaje aquí. Sé lo que no funcionó, y espero desarrollar aprendizajes en Queens para mejorar o modificar situaciones... Ahora estoy lista para aprender a hacer lo que ya se de mejor manera más dinámicamente. Heidi. (Featherstone, Munby y Russell, 1997: 94).
\end{abstract}

Las fortalezas y desafíos acerca de donde posicionar la práctica en relación a las clases de educación están resumidas en la Tabla 1. Esta tabla pone el acento en el asunto pedagógico central si es que la práctica viene tempranamente en el programa: ¿Qué pueden hacer los formadores de profesores en sus clases para ayudar a los estudiantes a aprender de su experiencia?

\title{
3.2.3. Trayendo las experiencias prácticas a los cursos universitarios
}

Qué fácil es escribir que "la tarea del formador de profesores es ayudar a los estudiantes a explorar y refinar sus percepciones". ¿Qué podría significar esto en la práctica, y de qué manera se relaciona con supuestos vinculados a la perspectiva de "práctica primero, comprensión después"? Durante los últimos 10 años, mi membresía en el grupo de SelfStudy de Prácticas de Formación Docente (S-STEP) - un grupo al interior de la American Educational Research Association- ha contribuido e inspirado mis propios esfuerzos por motivar el aprendizaje desde la experiencia en mi trabajo con profesores en formación inicial. Los puntos que vienen a continuación intentan resumir mi propio aprendizaje profesional y mi esfuerzo por comprender el significado de los puntos ya expuestos en las citas del trabajo de Kessels y Korthagen (1996). Korthagen et al. (2001) es una referencia fundamental en esta temática.

a) Escucha a los que están aprendiendo a enseñar. Mis propios estudiantes me han ayudado a aprender la mayor parte de lo que entiendo acerca de cómo incorporar sus experiencias en mi programa de enseñanza (Loughran y Russell, 1997). Munby y Russell (1994) presentan un argumento en términos de reconocer que hay una "autoridad de la experiencia" que es bastante nueva para la mayor parte de los estudiantes en formación y que requiere de ajustes apropiados en las clases del pregrado. Cook-Sather (2002) ha provisto la defensa más elaborada que hay hasta el momento a favor de escuchar a los que estamos 
enseñando; ayudar a que los profesores en formación exploren y refinen sus percepciones ciertamente requiere escucharlos y trabajar con ellos. De entre las temáticas habituales en un curso de didáctica de las ciencias, mis estudiantes y yo seleccionamos aquellas estrategias que resultan más urgentes a la luz de sus trasfondos y sus experiencias acumuladas.

Tabla 1. Respuestas ante asunciones sobre la práctica

\begin{tabular}{|c|c|c|}
\hline Pregunta & Primero teoría, luego práctica & Primero práctica, luego teoría \\
\hline Razones & $\begin{array}{l}\text { - Introducción gradual de la práctica es } \\
\text { utilizada comprensiblemente en los } \\
\text { campos donde el principiante es com- } \\
\text { pletamente inexperto. Los aspirantes a } \\
\text { médico no intervienen directamente en } \\
\text { una cirugía, ni tampoco un futuro abo- } \\
\text { gado interviene directamente en un juz- } \\
\text { gado. } \\
\text { - "Teoría primero, luego práctica" es el } \\
\text { enfoque habitual usado en la mayor } \\
\text { parte de las escuelas y las aulas } \\
\text { universitarias. } \\
\text { - Este enfoque persiste en parte porque } \\
\text { formadores de profesores quieren ser } \\
\text { útiles a aquellos que se supone que } \\
\text { saben tan poco sobre la enseñanza. } \\
\text { - Los formadores de docentes temen que } \\
\text { los nuevos maestros se 'contaminen' } \\
\text { por las prácticas escolares existentes. } \\
\text { - Suponemos, o al menos tenemos la } \\
\text { esperanza, que nuestras palabras pueden } \\
\text { proteger el inicio profesional de los } \\
\text { futuros profesores de la "enfermedad" } \\
\text { de malas las prácticas de enseñanza. }\end{array}$ & $\begin{array}{l}\text { - Aquellos que enseñan a enseñar siempre } \\
\text { informan que la práctica es el elemento } \\
\text { del programa más importante. } \\
\text { - 'Nadar o morir' parece drástico, pero si } \\
\text { se tiene éxito con la teoría primero, } \\
\text { también se tendrá éxito con la práctica } \\
\text { primero. } \\
\text { - Si queremos preparar a los nuevos } \\
\text { maestros con una predisposición para } \\
\text { mejorar lo que sucede en las escuelas, } \\
\text { comenzar con experiencia da un fuerte } \\
\text { mensaje de que un nuevo tipo de } \\
\text { aprendizaje es una parte importante de } \\
\text { aprender a enseñar. } \\
\text { - Si queremos ayudar a los nuevos } \\
\text { profesores a aprender a aprender de la } \\
\text { experiencia, entonces, partir de la } \\
\text { experiencia puede ser la mejor manera } \\
\text { de iniciar y apoyar ese objetivo. } \\
\text { - Los que aprenden a enseñar tiene muchas } \\
\text { ganas de aprender los misterios del } \\
\text { profesor viendo el primer día de escuela: } \\
\text { ¿Cómo se las arreglan para empezar las } \\
\text { cosas?” }\end{array}$ \\
\hline Reservas & $\begin{array}{l}\text { - La educación puede ser argumentada de } \\
\text { forma diferente de todas las otras } \\
\text { profesiones, porque todos los profesores } \\
\text { tiene más de } 15.000 \text { horas de experiencia } \\
\text { observando a los docentes hacer su } \\
\text { trabajo. } \\
\text { - 'Teoría primero' puede ser cuestionable } \\
\text { en enseñanza porque las imágenes (en } \\
\text { contexto) son más poderosas que las } \\
\text { palabras (escuchadas fuera de contexto). } \\
\text { - La tradición de la introducción gradual } \\
\text { puede ser en realidad una réplica de las } \\
\text { pobres prácticas que deseamos evitar. } \\
\text { - Adición de la práctica reflexiva a la } \\
\text { teoría primero "puede ser mal guiado". } \\
\text { - En un enfoque de "teoría primero", las } \\
\text { experiencias prácticas rara vez reciben } \\
\text { detallada atención en las clases } \\
\text { universitarias. }\end{array}$ & $\begin{array}{l}\text { - Los formadores de profesores parecen } \\
\text { guardar un cierto temor a que los } \\
\text { estudiantes lleguen a "enamorarse" a tal } \\
\text { punto de la práctica que pierdan todo } \\
\text { interés en lo "teórico". } \\
\text { - Colectivamente, los formadores de } \\
\text { docentes parecen tener un compromiso } \\
\text { con la idea de que los estudiantes deben } \\
\text { vincularse primero con el lugar de la } \\
\text { formación del profesorado. Menos unión } \\
\text { con el sitio practicum presumiblemente } \\
\text { sigue en algún momento posterior. } \\
\text { - Muchos enfoques formadores de } \\
\text { profesores parten de la suposición de que } \\
\text { ellos saben poco y necesitan que se les } \\
\text { diga mucho. } \\
\text { - Los maestros con experiencia pueden ser } \\
\text { reacios a que los nuevos maestros } \\
\text { observen los primeros días estresantes } \\
\text { del año escolar. }\end{array}$ \\
\hline
\end{tabular}


- El problema teórico-práctica persiste a través de todos los maestros.

- Tanto la teoría y la investigación tienden a ser vistos como irrelevantes.

Problemas

- Llamadas persistentes para la reforma escolar y mejora a menudo miran a los nuevos profesores, sin embargo, los nuevos maestros no se ven a sí mismos como agentes de cambio.

\footnotetext{
- Puede ser imposible para un gran grupo de formadores de docentes hacer un compromiso de mayor innovación.
}

b) Mostrarlas intenciones desde el principio y de manera frecuente. Cuando un estudiante con mucha experiencia en Aprendizaje Autodirigido (Self-directed Learning - SDL) sugirió que las experiencias con esa aproximación podrían ayudar a sustentar el aprendizaje desde la experiencia, me propuse probarlo. Encontré un libro de texto apropiado al respecto (Gibbons, 2002) y anuncié que dedicaríamos parte de nuestro tiempo a explorar una aproximación a SDL luego que hubieran ganado algo de experiencia enseñando. Para cuando comenzamos, los estudiantes no estaban sorprendidos; la mayoría estaban contentos con lo que habían aprendido acerca de ellos mismos, tanto en aspectos positivos como negativos. Al término del curso, decidieron poner las letras S D L al centro de uno de los paneles del techo de mi sala de clases.

c) Modela e interpreta, sin sermonear. ¿Por qué será que la frase "las acciones hablan más fuerte que las palabras" tan rara vez ha llegado a ser un principio para la enseñanza en los programas de formación inicial? ¿Por qué mis estudiantes continúan recibiendo clases expositivas acerca de por qué no deberían usar clases expositivas con sus estudiantes? Posiblemente se deba a que nuestra larga experiencia de sentarnos a escuchar lo que alguien dice, nos lleva a enseñar del mismo modo en que fuimos enseñados. Segall (2002) argumenta de manera contundente que necesitamos crear experiencias en las clases de formación inicial que ilustren los principios que queremos "transmitir" a los nuevos profesores. Kroll (2004) ofrece una descripción muy clara acerca de cómo logró operacionalizar en su propia enseñanza un set de principios fundamentales del programa, escribiendo que "el caso ilustra cómo el modo en que a los futuros profesores se les enseña teoría puede ayudarlos a integrar sus propias ideas acerca del aprendizaje y la enseñanza con teoría constructivista, para pensar críticamente acerca de sus propias prácticas en una modalidad de desarrollo continuo" (2004: 199). Casi inconscientemente, hace poco me sorprendí a mí mismo "enseñando" la significatividad de comportamientos no verbales, pero sin decirles a los estudiantes que el tiempo que un profesor guarda silencio puede hacer la diferencia en la manera en que los estudiantes responden a las preguntas. En lugar de ello, me quedé en silencio deliberadamente por mucho más tiempo de lo habitual, para luego explicarles lo que estaba haciendo. El impacto fue dramáticamente distinto; estaban fascinados de aprender que los profesores pueden lograr una enseñanza productiva sin usar palabra alguna.

\subsubsection{Enseñar a reflexionar de modo indirecto}

¿Con cuanta frecuencia los estudiantes escuchan que deberían volverse "profesionales críticos y reflexivos"? ¿Qué significado posible podrían llegar a tener esas palabras para alguien con poca o nula experiencia en la enseñanza? El año 2001, uno de mis estudiantes me sugirió que parara de "empujar" la reflexión, y en lugar de ello enseñara a reflexionar, 
y luego mostrara qué y cómo lo había hecho. Ya no ocupo más la "palabra prohibida" (reflexión) en mis clases. En lugar de ello, con muy poca explicación de por medio le entrego a cada estudiante un documento, una tabla en la que tienen que ir respondiendo preguntas abiertas acerca de cinco etapas de su progreso a través de nuestro programa:

a. Después de tres semanas de clases

b. Después de las primeras cinco semanas de práctica

c. Después de dos semanas más de clases y cinco semanas más de práctica

d. Después de siete semanas más de clases

e. Después de seis semanas más de práctica y dos semanas finales de clases

Una tabla de tres columnas es crucial para ayudar a los candidatos a tener algún sentido con respecto a sus experiencias, como se muestra en la Tabla 2 (usada con permiso del autor). La segunda columna provee un espacio reducido inicialmente que se expande para contener toda la información que ellos quieran incluir; la tercera columna da espacio para mis comentarios inmediatamente al lado de los de ellos. Cuando tienen que revisar todo lo que han puesto, como parte del trabajo del curso, se dan cuenta de cuanto han cambiado sus visiones a medida que ganaban experiencia. También parecen valorar mis extensos comentarios, lo que resulta ser una alternativa poco usual frente a los habituales comentarios cortos en los márgenes de un trabajo. Con las cinco tablas completadas, el escenario queda preparado para finalizar el curso con la identificación de sus escritos como "reflexiones" y recordar la manera en que ese tipo de escritura los ayuda a vincular el cambio de perspectiva en sus acciones pedagógicas.

Tabla 2. Diálogo estudiante en práctica formador

\begin{tabular}{|c|c|c|}
\hline Pregunta & Respuesta futuro profesor & Comentario del formador \\
\hline \multirow[t]{3}{*}{$\begin{array}{l}\text { ¿Qué nuevas metas } \\
\text { y puntos de vista te } \\
\text { han proporciona- } \\
\text { do, las clases en } \\
\text { enero-febrero para } \\
\text { tu desarrollo como } \\
\text { profesor? }\end{array}$} & $\begin{array}{l}\text { Yo pienso que las clases de enero de hecho me } \\
\text { han mostrado que el desarrollo profesional y mi } \\
\text { desarrollo como profesor, se pueden potenciar. } \\
\text { Yo seré el primero en admitir que yo no era una } \\
\text { persona muy filosófica cuando entré a este } \\
\text { programa, pero pienso que podría ser, además, el } \\
\text { primero en admitir que me he convertido en una. } \\
\text { Pienso que un importante aprendizaje ha sido } \\
\text { darse cuenta que los objetivos y el desarrollo son } \\
\text { bastante tangibles. }\end{array}$ & $\begin{array}{l}\text { Usted ha hecho esto mucho más } \\
\text { rápido e impresionantemente que } \\
\text { nadie que yo pueda recordar! } \\
\text { Y fue un placer verlo porque además } \\
\text { yo ya había visto lo BUENAS que ya } \\
\text { eran tus prácticas de enseñanza. } \\
\text { Tienes el coraje para poner en acción } \\
\text { tus convicciones y luego ver cómo } \\
\text { tus estudiantes responden. De eso se } \\
\text { trata la enseñanza! }\end{array}$ \\
\hline & $\begin{array}{l}\text { Vuelvo a pensar en José "Cómo yo tuve éxito en } \\
\text { McArthur", 'y uno de sus puntos era, 'Permítete a } \\
\text { mí mismo cambiar. 'Eso es exactamente lo que } \\
\text { tienes que hacer". No pongas esfuerzo en esto, esto } \\
\text { simplemente sucede. Deja de preocuparte o sobre } \\
\text { analizarlo. Cuándo dejas de hacer estas cosas, o } \\
\text { poner demasiado esfuerzo, el cambio empieza. }\end{array}$ & $\begin{array}{l}\text { EXACTAMENTE es el enlace } \\
\text { correcto que debe hacerse en este } \\
\text { punto. (Tal vez deba ponerlo en } \\
\text { nuestro salón de clases junto a las } \\
\text { otras pequeñas señales que ya hay) }\end{array}$ \\
\hline & $\begin{array}{l}\text { Debo decir que esto se siente increíble. } \\
\text { Ese es uno de los más poderosos consejos que he } \\
\text { recibido este año, y si puedo enviar un mensaje a } \\
\text { cualquier persona del próximo año, sería lo } \\
\text { mismo. } \\
\text { No recibo ningún crédito por decirlo a la clase } \\
\text { del próximo año, pero es increíble cuando te das } \\
\text { cuenta de que ha sucedido. }\end{array}$ & $\begin{array}{l}\text { BIEN! } \\
\text { ¿Alguna idea sobre cómo y cuándo } \\
\text { introducirlo? } \\
\mathrm{Si} \text { hubiera hablado contigo en } \\
\text { septiembre, es posible que hubieses } \\
\text { tenido un signo de pregunta en tu } \\
\text { rostro! }\end{array}$ \\
\hline
\end{tabular}


a) Reconocer que transmitir es lo más cómodo. Muchos candidatos a profesor que llegan a los programas de pedagogía para profesionales, lo hacen con la expectativa que aprenderán a enseñar de la misma manera en que aprendieron sus materias en la formación de pregrado. Tienden a asumir que exponer algo en un discurso produce aprendizaje profesional. Tienden a asumir que saben más acerca de la materia que enseñarán de lo que sus estudiantes saben, y frecuentemente piensan que necesitan que alguien les cuente: 1) cómo planificar una clase y una unidad, y 2) cómo manejar la disciplina de los estudiantes en la sala de clases. También creen que la tarea de un profesor es "pasar" el currículum, y que para eso es necesario saberse todas las respuestas a las preguntas que los estudiantes podrían llegar a hacer. Aunque trato de no criticar esas creencias previas de manera explícita, trabajo de manera subliminal en mi enseñanza para revertir esas creencias, siempre de manera constructiva para que la comodidad de la transmisión se vea reemplazada no por la incomodidad, sino por la comodidad de aprender de la experiencia.

b) Buscar coherencia y consistencia por sobre todo. Todo cambio en la enseñanza es difícil y desafiante; todos tenemos la fuerte tendencia a volver a estrategias que nos resultan más familiares y cómodas, como la de "decirle" cosas a los estudiantes, especialmente cuando "hay poco tiempo y tanta materia por pasar". No hay nada que interfiera más con nuevas aproximaciones y mensajes como las inconsistencias e incoherencias. Una vez que uno comienza una serie de nuevas prácticas que implican nuevas perspectivas acerca de cómo las personas aprenden a enseñar (como por ejemplo, explorar el impacto de las experiencias de práctica y del programa), la coherencia y consistencia para desarrollar esas nuevas prácticas se vuelven parte esencial del proceso para alcanzar los efectos esperados.

Mi aprendizaje en los últimos 10 años ha sido el más emocionante de mis 25 años en la formación inicial de profesores. Hay mucha coherencia y consistencia en un grupo de supuestos acerca de la experiencia como algo primario, particularmente, si se logra reconocer a los supuestos de "teoría primero" como inadecuados. Sin embargo, también hay mucho de desafío (e incertidumbre y confusión) al trabajar para operacionalizar un nuevo set de ideas, del mismo modo que presenta un desafío para aquellos que están aprendiendo a enseñar y quieren mejorar la calidad del aprendizaje de sus estudiantes.

\section{A MODO DE CONCLUSIONES}

Al escribir acerca de los docentes y el conocimiento técnico en "especulaciones de cambio" al final de su estudio, Lortie (1975) refiere a asuntos que bien podrían aplicarse a la predisposición que tienen los formadores de profesores para evitar la consideración de supuestos fundamentales acerca de la práctica:

El ethos del quehacer tiene una inclinación contraria al involucramiento en indagación pedagógica. El conservadurismo reflexivo implícitamente niega la significatividad del conocimiento técnico, asumiendo que las energías deberían concentrarse en la realización de objetivos convencionales y formas conocidas. El individualismo lleva a desconfiar del concepto de saber compartido [sic]; presenta la enseñanza como la expresión de una personalidad individual. Las orientaciones presentistas retardan el hacer sacrificios ahora para lograr beneficios después; la indagación descansa en un valor opuesto a ese. (Lortie, 1975: 240). 
Las ideas acerca del lugar de la práctica en un programa de formación inicial docente se encuentran estrechamente entretejidas con asuntos relacionados a la reforma de la formación de profesores y con el mejoramiento escolar. No debería sorprendernos que los asuntos de calidad en el aprendizaje en la escuela y la calidad del aprendizaje profesional en las universidades comparten una misma base. Reafirmo aquí que los supuestos acerca del uso de los tiempos y las estructuras de los programas de formación docente se han mantenido sin ser examinados en los contextos de reforma educativa. Surge así una serie de preguntas que se podría hacer a los formadores de profesores:

¿Estamos preparados para repensar la estructura de la formación inicial, de manera de reducir la brecha que los nuevos profesores perciben entre teoría y práctica?, ¿Estamos preparados para repensar la relación entre la práctica y los cursos que enseñamos?, ¿Estamos preparados para repensar la naturaleza del aprendizaje desde la experiencia, para comprender la potencial contribución de la phronesis al aprendizaje profesional? ¿Estamos preparados para repensar nuestras acciones en los programas y cursos de formación docente, si nuestras reflexiones indican que hay potencial para el mejoramiento de la calidad del aprendizaje en la formación docente?

Los vínculos estructurales entre teoría y práctica parecen estar ausentes en muchos programas de formación de profesores, y las explicaciones para ello podrían incluir algo de lo siguiente:

Los que están aprendiendo a enseñar ya tienen opiniones bastante firmes acerca de lo que podría constituir un vínculo entre teoría y práctica. Esas opiniones tienden a ser bastante improductivas, y es muy posible que la experiencia de práctica en la escuela termine reafirmando esas ideas en lugar de desafiarlas.

Los formadores de formadores tienen ideas igualmente firmes e improductivas acerca de los vínculos entre teoría y práctica, o entre escuela y universidad. Quizás sin la intención de hacerlo, muchos formadores de profesores parecen seguir la postura tradicional de que la teoría es algo que debe enseñarse primero y que la práctica viene después. Esta visión tradicional está profundamente arraigada tanto en la epistemología de la universidad como en la organización curricular de la escuela.

Para resumir, la manera en que aprendemos de la experiencia continúa siendo una temática descuidada y escasamente comprendida, a pesar de su relevancia para la reforma y la mejora. Tanto en los contextos de escuela como de formación docente, las perspectivas de investigación sobre cambio conceptual y aprendizaje autodirigido tienen enormes implicancias en la manera en la que enseñamos. Extender estas perspectivas hacia la estructura de los elementos de la práctica y nuestros supuestos acerca de ella podría proveer una ayuda invaluable en el proceso de mejoramiento conjunto tanto de la escuela como de la formación docente.

\section{REFERENCIAS BIBLIOGRÁFICAS}

Bruner, J.S. (1996). The culture of education. Cambridge, MA: Harvard University Press.

Cook-Sather, A. (2002). Authorizing students' perspectives: Toward trust, dialogue, and change in education. Educational Researcher, vol.31, n.4, 3-14.

Featherstone, D., Munby, H., \& Russell, T. (1997). Finding a voice while learning to teach. London, UK: Falmer Press. 
Gibbons, M. (2002). The self-directed learning handbook: Challenging adolescent students to excel. San Francisco, CA: Jossey-Bass.

Goodlad, J.I. (1990). Teachers for our nation's schools. San Francisco, CA: Jossey Bass.

Kessels, J., \& Korthagen, F. (1996). The relationship between theory and practice: Back to the classics. Educational Researcher, vol.25, n.3, 17-22.

Korthagen, F., \& Kessels, J. (1998). Linking theory and practice: Changing the pedagogy of teacher education. Educational Researcher, vol.28, n.4, 4-17.

Korthagen, F., Kessels, J., Koster, B., Lagerwerf, B., \& Wubbels, T. (2001). Linking practice and theory: The pedagogy of realistic teacher education. Mahwah, NJ: Lawrence Erlbaum Associates.

Kroll, L. R. (2004). Constructing constructivism: How student-teachers construct ideas of development, knowledge, learning and teaching. Teachers and Teaching: Theory and Practice, vol.10, n.2, 199-221.

Lortie, D. C. (1975). Schoolteacher: A sociological study. Chicago, IL: University of Chicago Press.

Loughran, J., \& Russell, T. (1997). Meeting student teachers on their own terms: Experience precedes understanding. In V. Richardson (Ed.), Constructivist teacher education: Building a world of new understandings (pp. 164-181). London, UK: Falmer Press.

MacDonald, B. (1975). Introduction. In J. Elliott \& B. MacDonald (Eds.), People in classrooms (pp. 2-13). Norwich, UK: Centre for Applied Research in Education, University of East Anglia.

Munby, H., \& Russell, T. (1994). The authority of experience in learning to teach: Messages from a physics methods class. Journal of Teacher Education, vol.45, n.2, 86-95.

Russell, T. (1999). The challenge of change in teaching and teacher education. In J. R. Baird (Ed.), Reflecting, teaching, learning: Perspectives on educational improvement (pp. 219-238). Cheltenham, Victoria: Hawker Brownlow Education.

Russell, T. (2000, July). Moving beyond 'default' teaching styles and programme structures: The rise, fall, and marginal persistence of reflective practice in preservice teacher education in the period 1984-2000. Paper presented at the First Carfax International Conference on Reflective Practice, Worcester, England.

Sarason, S. B. (1971). The culture of the school and the problem of change. Boston, MA: Allyn \& Bacon.

Sarason, S. B. (1993). The case for change: Rethinking the preparation of educators. San Francisco, CA: Jossey-Bass.

Sarason, S. B. (1996). Revisiting 'The culture of the school and the problem of change.' New York, NY: Teachers College Press.

Segall, A. (2002). Disturbing practice: Reading teacher education as text. New York, NY: Peter Lang. 